\title{
Functional Role of a Protein Foldon-An $\Omega$-Loop Foldon Controls the Alkaline Transition in Ferricytochrome c
}

\author{
Haripada Maity, * Jon N. Rumbley, and S. Walter Englander \\ The Johnson Research Foundation, Department of Biochemistry and Biophysics, University of Pennsylvania School of Medicine, \\ Philadelphia, Pennsylvania
}

\begin{abstract}
Hydrogen exchange results for cytochrome c and several other proteins show that they are composed of a number of foldon units which continually unfold and refold and account for some functional properties. Previous work showed that one $\Omega$-loop foldon controls the rate of the structural switching and ligand exchange behavior of cytochrome c known as the alkaline transition. The present work tests the role of foldons in the alkaline transition equilibrium. We measured the effects of denaturant and 14 destabilizing mutations. The results show that the ligand exchange equilibrium is controlled by the stability of the same foldon unit implicated before. In addition, the results obtained confirm the e-amino group of Lys79 and Lys73 as the alkaline replacement ligands and bear on the search for a triggering group. Proteins 2006;63:349-355. ๑ 2005 Wiley-Liss, Inc.
\end{abstract}

Key words: cytochrome c; hydrogen exchange; alkaline transition; foldon

\section{INTRODUCTION}

Hydrogen exchange (HX) results have revealed a previously unsuspected aspect of protein structure and dynamical behavior. Some proteins, perhaps many, are composed of a number of structural units called foldons which continually unfold and refold in a concerted way even under native conditions. ${ }^{1}$ It appears that the behavior of foldon units can contribute to a number of protein properties and functions. Most strikingly, foldon behavior constructs stepwise protein unfolding and refolding pathways. ${ }^{1-7}$ Further, results for cytochrome c (Cyt c) connect its foldon units with some other functional behaviors. ${ }^{8,9}$ One of these is the structural switching reaction that exchanges alternative heme ligands at elevated $\mathrm{pH}$, known as the alkaline transition.

Figure 1 depicts equine Cyt c, a moderately small protein with 104 amino acids, three major helices, three large $\Omega$-loops, and a covalently bound heme group. The color coding in Figure 1 shows the five cooperative foldon units that compose Cyt c. In the alkaline transition, the Met80-S ligand, which is weakly coordinated to the heme iron, is replaced ${ }^{10}$ by a nearby lysine $\epsilon$-amino group. ${ }^{11,12}$ The normal heme ligand (Met80) and the candidate replacement ligands (Lys72, 73, and 79) are all placed in the red loop foldon, a 16-residue $\Omega$-loop (residue 70 to 85 ).

Previous HX results indicated that the rate of the alkaline transition, reached at high $\mathrm{pH}$, is limited by the rate at which the red $\Omega$-loop foldon transiently unfolds. ${ }^{8}$ The present work investigated the role of Cyt $\mathrm{c}$ foldons in the alkaline transition equilibrium.

\section{Materials}

\section{MATERIALS AND METHODS}

$\mathrm{D}_{2} \mathrm{O}(99.9 \%)$ was from Sigma-Aldrich, guanidinium chloride $(\mathrm{GdmCl})$, and guanidinium thiocyanate (GdmSCN) were from ICN. Deuterated GdmSCN was prepared by dissolving in $\mathrm{D}_{2} \mathrm{O}$ and lyophilizing three times. Protein concentration was measured using an extinction coefficient of $106 \mathrm{mM}^{-1} \mathrm{~cm}^{-1}$ at $409 \mathrm{~nm}$ for oxidized Cyt c. ${ }^{13}$ Refractive index measurements were used to determine the concentration of $\mathrm{GdmCl}^{14}$ and $\mathrm{GdmSCN} .{ }^{15}$ Other chemicals were from Sigma and Fisher.

\section{Recombinant pWT Cyt c}

The present experiments used a recombinant Cyt c based on the construct developed by Pollock et al. ${ }^{16}$ with coexpression of the yeast heme lyase. The yeast sequence was changed to horse, optimized for expression in Escherichia coli, and purified as described before. ${ }^{17}$ The recombinant protein, referred to as pseudo wild type (pWT) Cyt c, has the equine Cyt $\mathrm{c}$ sequence except for the replacement of two peripheral histidines (His26 and 33 to Asn) and the absence of $\mathrm{N}$-terminal acetylation. Due to the absence of the normal internally H-bonded His26, pWT equine Cyt is slightly less stable than the wild type (WT) protein at pH 7 and above and more stable at lower pH. Its alkaline transition $\mathrm{pK}_{\mathrm{a}}$ is 9.16 compared to 9.42 in WT Cyt c. Constructs in which lysines in the red $\Omega$-loop foldon were mutated also contain the mutation Val3Pro intended to increase global stability. ${ }^{18}$

For each mutational variant and denaturant concentration, experiments were done to measure the $\mathrm{pK}_{\mathrm{a}}$ of the

Abbreviations: Cyt c, cytochrome c; pWT, pseudo wild type; HX, hydrogen exchange; GdmSCN, guanidinium thiocyanate; GdmCl, guanidinium chloride; AT, alkaline transition

Jon N. Rumbley's present address is Department of Chemistry, University of Minnesota, 313 Chemistry, 1039 University Dr., Duluth, MN 55812.

*Correspondence to: Haripada Maity, The Johnson Research Foundation, Department of Biochemistry and Biophysics, University of Pennsylvania School of Medicine, Philadelphia, Pennsylvania 19104. E-mail: hmaity@mail.med.upenn.edu

Received 23 May 2005; Revised 29 July 2005; Accepted 31 July 2005

Published online 14 November 2005 in Wiley InterScience (www.interscience.wiley.com). DOI: 10.1002/prot.20756 


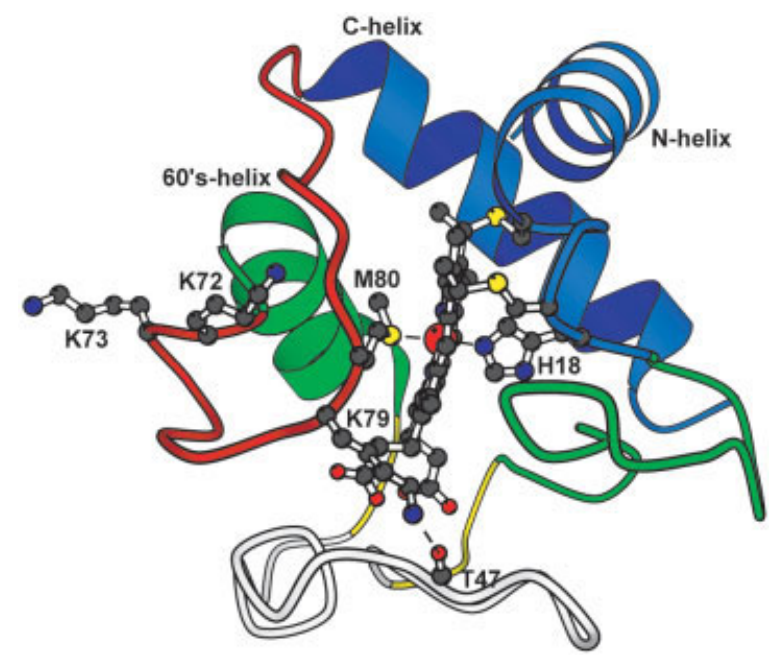

Fig. 1. Diagram of Cyt c structure (Molscript ${ }^{37}$ ). The normal Met80 heme ligand and the lysine misligation candidates, all in the red loop, are shown. The color coding shows the foldon units identified in native state $\mathrm{HX}$ experiments. ${ }^{2,9}$ The present work focusses on the role of the red $\Omega$-loop foldon in the alkaline transition.

alkaline transition ( $\mathrm{pH}$ titration), the global protein stability (denaturant melting), and the stability of the red loop foldon (HX rate). All experiments were done at $20^{\circ} \mathrm{C}$ with $\mathrm{KCl}$ added to maintain ionic strength at $0.5 \mathrm{M}$.

\section{Alkaline Transition}

The alkaline transition $\mathrm{pK}_{\mathrm{a}}$ was measured by manual $\mathrm{pH}$ titration monitored by the amplitude of the charge transfer absorption band at $695 \mathrm{~nm}$, which is specific for the Met80-S to heme Fe ligation. The native Cyt c structure resists the transition so that factors that destabilize the responsible parts of the native structure promote the transition. Change in structural stability against the alkaline transition due to denaturant or mutation was measured in terms of changes in the $\mathrm{pK}_{\mathrm{a}}$ and calculated as in Equation (1), written for a one proton titration.

$$
\Delta \Delta \mathrm{G}_{\mathrm{AT}}^{\circ}=-\mathrm{RT} \Delta \ln \mathrm{K}_{\mathrm{AT}}=2.303 \mathrm{RT} \Delta \mathrm{pK}_{\mathrm{AT}}
$$

The index AT refers to the alkaline transition, $\mathrm{R}$ is the universal gas constant, and $\mathrm{T}$ is absolute temperature.

\section{Protein Stability}

Global protein stability was measured by denaturant melting (both fluorescence and circular dichroism; Aviv 202 CD spectrometer with automated pipettor). Linear extrapolation ${ }^{19}$ of the two-state fitting equation ${ }^{20}$ to zero denaturant yields $\Delta G^{o}$ values that are too low because Cyt c equilibrium melting is not fully two-state ${ }^{21}$; partially unfolded intermediates are significantly populated in the transition zone. In such cases, the midpoint value is minimally affected but the slope $(\mathrm{m}=\mathrm{d} \Delta \mathrm{G} \% \mathrm{~d}$ [denaturant]) is reduced ${ }^{21,22}$ and leads to an artificially low value for $\Delta G^{\circ}$. Nevertheless a comparison of melting results and HX results for multiple constructs shows that this approach fairly accurately measures the change in stability $\left(\Delta \Delta G^{\circ}\right)$ due to the mutants used here.

\section{Foldon Stability}

The stability of the different Cyt $\mathrm{c}$ foldons was obtained by measuring the HX rates of marker protons ( $\mathrm{pDr} 7$, $20^{\circ} \mathrm{C}$ ). Marker protons are those that exchange only when the entire foldon that contains them transiently unfolds. $\mathrm{H}$ to $\mathrm{D}$ exchange was measured by placing samples in $\mathrm{D}_{2} \mathrm{O}$ and recording 2D COSY spectra over time. HX rates were converted to stabilization free energy by Equation 2 .

$$
\Delta \mathrm{G}^{\mathrm{o}}(\mathrm{HX})=-\mathrm{RT} \ln \mathrm{K}_{\mathrm{op}}=-\mathrm{RT} \ln \left(\mathrm{k}_{\mathrm{int}} / \mathrm{k}_{\mathrm{ex}}\right)
$$

where $\mathrm{k}_{\mathrm{ex}}$ is the measured HX rate and $\mathrm{k}_{\mathrm{int}}$ is the intrinsic HX rate expected for each amide proton when protecting structure is absent. ${ }^{23,24}$ For HX as a function of added denaturant, we used the strong denaturant, GdmSCN, to minimize the deleterious effect of higher ionic strength on NMR spectra.

\section{RESULTS}

In order to investigate the possible role of the several Cyt $\mathrm{c}$ foldons in the alkaline transition, we measured the alkaline transition $\mathrm{pK}_{\mathrm{a}}$ as in Figure 2(A), the stability of the different foldons as in Figure 2(B), and global protein stability by denaturant melting (Table I). Stability was varied in a general way by addition of denaturant and in a more specific way by mutations placed at known positions throughout the protein. For each mutation and each denaturant concentration, the change in stability against the alkaline transition was compared with the change in global and foldon stabilities. Results are listed in Table I and shown in Figure 2.

\section{The Red Loop Foldon Controls the Alkaline Transition}

The $\mathrm{pH}$ titration curves in Figure 2(A) measure the alkaline transition for pWT Cyt $\mathrm{c}$ in increasing concentrations of GdmSCN. The transition has $\mathrm{pK}_{\mathrm{a}} 9.16$ in the absence of denaturant and it is promoted by increasing denaturant $\left(\mathrm{pK}_{\mathrm{a}}\right.$ decreases). This behavior has previously been attributed to some general protein destabilization. HX results in Figure 2(B) show the effect of denaturant on the stability of the different Cyt $\mathrm{c}$ foldon units measured at pDr 7 and $20^{\circ} \mathrm{C}$, well below the alkaline transition. The stability of each foldon is represented by a marker proton, the exchange rate of which is determined by the reversible unfolding of the foldon that exposes it to exchange. ${ }^{2}$ The $\mathrm{HX}$ rate of each marker proton was converted to the $\Delta \mathrm{G}_{\mathrm{HX}}^{\circ}$ for unfolding of its parent foldon by use of Equation 2 .

The foldon units increase in stability $\left(\Delta \mathrm{G}_{\mathrm{HX}}^{\mathrm{o}}\right)$ in the order red, yellow, green, and blue [Fig. 2(B)]. Their sensitivity to denaturant, indicated by the slope of the stability curves in Figure $2(B)$ (slope $=m=d \Delta G_{H X} / d$ [denaturant]), increases in the same order. The $\mathrm{m}$ value relates to the amount of molecular surface that is newly exposed in each transient unfolding reaction. ${ }^{25}$ The ladder of increasing free energy and size is explained by the fact that the foldons unfold in a sequence in which each higher lying unfolding includes all of the lower lying unfoldings. That is, the ladder reflects a sequential stepwise unfolding and refolding pathway with the steps determined by the foldon units. ${ }^{1}$ 

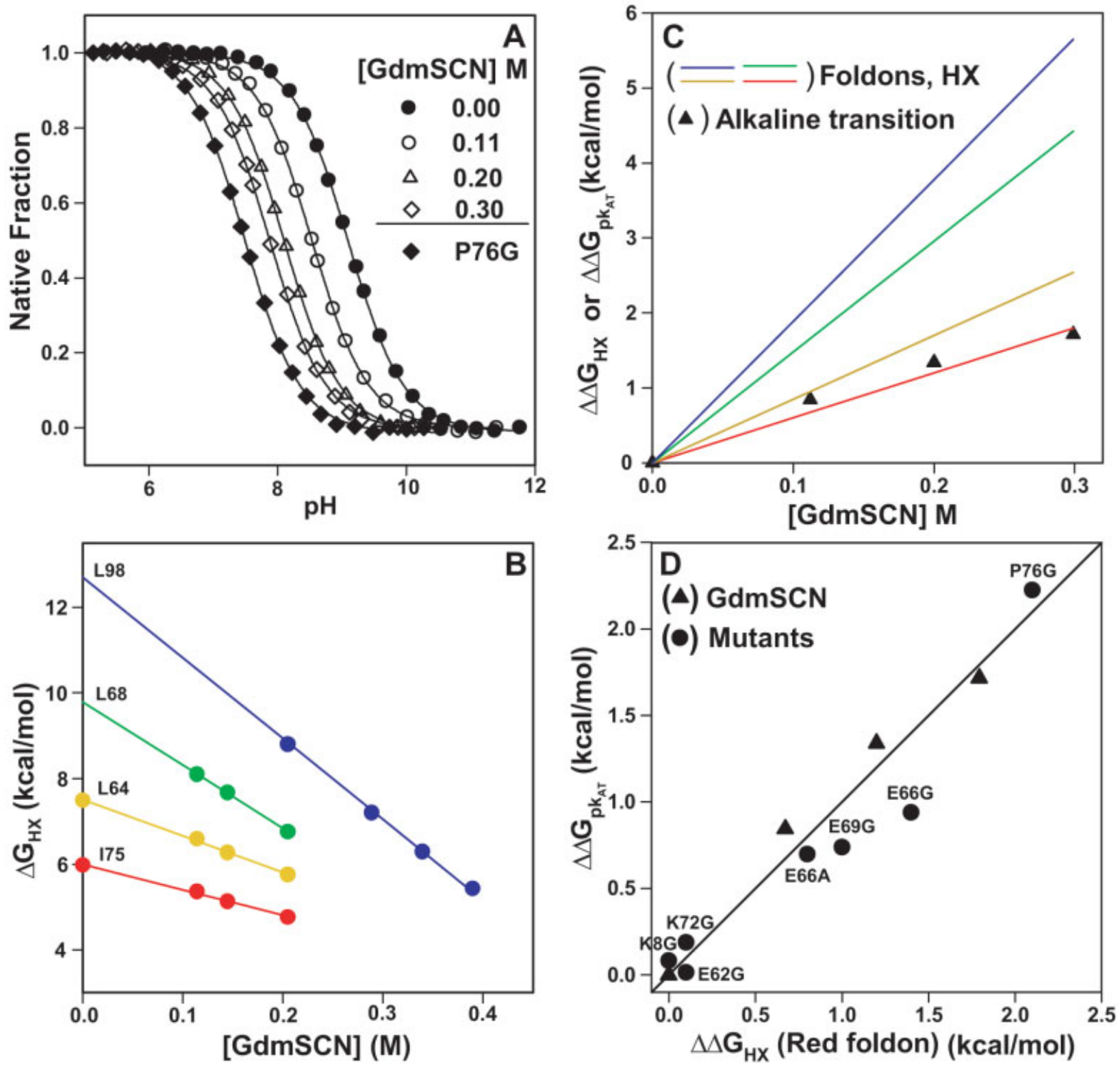

Fig. 2. Change in stability due to denaturant and mutations. A: The alkaline transition of recombinant pWT equine Cyt $\mathrm{c}$ measured by $695 \mathrm{~nm}$ absorbance (in $0.5 \mathrm{M} \mathrm{KCl}$ ). The $\mathrm{pK}_{\mathrm{a}}$ is 9.16 in zero denaturant and it decreases with added denaturant and with destabilizing mutations (Pro76Gly shown as an example). The fit [Eq. (1)] demonstrates a single proton transition; $\mathrm{pK}_{\mathrm{a}}$ values were reproducible to $\pm 0.03 \mathrm{pH}$ units. B: Stability of the Cyt c foldons measured by $\mathrm{H}$ to $\mathrm{D}$ exchange of amide marker protons (color coded), which exchange only when the foldon carrying them transiently unfolds (data from Maity et al. ${ }^{15}$ ). HX rates measured by NMR as a function of denaturant are displayed in terms of the free energy of the reversible equilibrium unfolding reaction that determines exchange [Eq. (2)]. The lowest lying nested-yellow $\Omega$-loop shown in Figure 1 exchanges too rapidly to measure under the present conditions. C: The alkaline transition and protein stability. Lines color coded as in Figure 1 show the denaturant-dependent change in stability of the different Cyt c foldon units, replotted from the HX data in part B. Triangles show the denaturant-dependent change in stability against the alkaline transition $\left(\mathrm{pK}_{\mathrm{AT}}\right)$ measured by $\mathrm{pH}$ titration as in panel $\mathrm{A}$. $\mathbf{D}$ : Change in stability against the alkaline transition $\left(\mathrm{pK}_{\mathrm{AT}}\right)$ compared to the change in stability of the red loop foldon. Stability was manipulated by denaturant (triangles) and mutations (circles). The line of unit slope is shown for comparison.

Figure 2(C) replots Figure 2(B) to more directly compare the effect of denaturant on the stability of the different foldons $\left(\Delta \Delta \mathrm{G}_{\mathrm{HX}}\right)$ with the effect on the alkaline transition $\left(\Delta \Delta \mathrm{G}_{\mathrm{pK}_{\mathrm{AT}}}\right)$. The destabilization of the alkaline transition is quantitatively identical to the destabilization of the red $\Omega$-loop foldon and not to the other foldons. We further tested the effects of destabilizing mutations placed at various positions throughout the Cyt c protein. Results are listed in Table I. Figure 2(D) compares the destabilization of the red loop and the alkaline transition for all of the mutants for which red loop stability was measured, and for the denaturant results just described.

The mutation Pro76Gly in the red loop destabilizes the red foldon by $2.1 \mathrm{kcal} / \mathrm{mol}$. It promotes the alkaline transition by this same amount (in agreement with Nall et al. ${ }^{26}$ for yeast iso-2-Cyt c). Several mutations placed in the green helix remove stabilizing cross-foldon $\mathrm{H}$-bonds to the red $\Omega$-loop (Glu66Gly, Glu66Ala, Glu69Gly). They destabi- 
TABLE 1. Measured Parameters

\begin{tabular}{lrrrr}
\hline Protein & \multicolumn{3}{c}{$\begin{array}{c}\Delta \Delta \mathrm{G} \\
\text { (global) }\end{array}$} & $\begin{array}{c}\Delta \Delta \mathrm{G}_{\mathrm{AT}} \\
\text { (red foldon) }\end{array}$ \\
\hline WT & 9.42 & -0.5 & -0.4 & -0.1 \\
pWT & 9.16 & 0.0 & 0.0 & 0.0 \\
E4G & 9.15 & 0.5 & 0.0 & N.D. \\
K8G & 9.10 & 0.5 & 0.1 & 0.0 \\
K22G & 9.23 & 1.7 & -0.1 & N.D. \\
K25G & 9.27 & 0.9 & -0.1 & N.D. \\
K39G & 9.18 & 0.7 & 0.0 & N.D. \\
E62G & 9.15 & 0.7 & 0.0 & 0.1 \\
E66G & 8.46 & 1.9 & 0.9 & 1.4 \\
E66A & 8.64 & 0.6 & 0.7 & 0.8 \\
E69G & 8.61 & 1.4 & 0.7 & 1.0 \\
P76G & 7.50 & 2.3 & 2.2 & 2.1 \\
K72G & 9.02 & 0.0 & 0.2 & 0.1 \\
K72,73G & 9.00 & 0.8 & 0.2 & N.D. \\
V3PK72QK73AK79R & 9.80 & $\approx-0.2$ & -0.9 & N.D. \\
$\quad$ Free N-terminus) & & & & \\
V3PK72QK73AK79R & 10.30 & N.D. & -1.5 & N.D. \\
(Blocked N-terminus) & & & & \\
\hline
\end{tabular}

Data are for WT Cyt c, pWT Cyt c, and pWT mutants. The alkaline transition $\mathrm{pK}_{\mathrm{a}}$ was measured by $\mathrm{pH}$ titration, global free energy by $\mathrm{GdmCl}$ melting using both $\mathrm{CD}$ and fluorescence, and foldon free energy by $\mathrm{HX}$ (zero denaturant). $\Delta \Delta \mathrm{G}$ is in $\mathrm{kcal} / \mathrm{mol}$. Conditions were $\mathrm{pH} 7.0,20^{\circ} \mathrm{C}$ except for $\mathrm{P} 76 \mathrm{G}$ which was measured at $\mathrm{pH} 5.0$ and compared to $\mathrm{pWT}$ at $\mathrm{pH} 5.0$.

lize the red foldon and the alkaline transition approximately equally. The mutation Lys72Gly in the red loop has a very small effect on red loop stability and on the alkaline transition.

To distinguish the possible role of the other Cyt $\mathrm{c}$ foldons in the alkaline transition, they were similarly destabilized by specifically placed mutations (Table I). Two mutations placed in the blue foldon, Glu4Gly and Lys8Gly in the $\mathrm{N}$-terminal helix, destabilize the protein by $0.5 \mathrm{kcal} / \mathrm{mol}$ but they have no effect on the alkaline transition or on the red loop stability (only Lys8Gly was measured). Two mutations placed in the green loop, Lys22Gly and Lys25Gly, destabilize the protein by about 2 and $1 \mathrm{kcal} /$ mol respectively but they have no effect on the alkaline transition (red loop test not done). Two mutations, Lys39Gly and Glu62Gly, destabilize the yellow neck foldon by $0.7 \mathrm{kcal} / \mathrm{mol}$ but they have no effect on the alkaline transition or on the red loop stability (only Glu62Gly was measured). These mutations do not affect the red loop foldon because they are placed in foldons that lie higher on the free energy unfolding ladder. ${ }^{1,27}$

In summary, denaturant destabilizes the red $\Omega$-loop and the alkaline transition equally whereas the other foldons are affected very differently. Similarly, mutations that destabilize the red loop destabilize the alkaline transition nearly equally whereas mutations that affect other foldons but not the red loop do not affect the alkaline transition. Thus the stability of the red $\Omega$-loop against unfolding appears quantitatively in the protein machinery that stabilizes against the alkaline transition, and this effect is specific for the red loop. These results are consistent with the fact that Met80 and the replacement lysines are all in the red loop, and it meshes with a previous result which showed that the limiting rate of the alkaline transition, reached at high $\mathrm{pH}$, is equal to the rate for red loop unfolding. ${ }^{8}$

\section{The Replacement Residues in the Alkaline Transition}

Other results obtained in this study relate to the amino acid residues that participate in the alkaline transition. The $\mathrm{pK}_{\mathrm{a}}$ of the high $\mathrm{pH}$ transition is generally consistent with lysine and also tyrosine, but the heme iron in the alkaline form is low spin, pointing to a nitrogen ligand. Among the 19 lysine residues in equine Cyt $\mathrm{c}$, the most obvious candidates, close to the normal Met80 ligand, are lysines 72, 73, and 79 in the red loop and lysines 86, 87, and 88 placed just after it. A comprehensive analysis of the literature together with additional penetrating work identified Lys73 and 79 as the alkaline ligands in yeast iso-1-Cyt c. ${ }^{11,12}$

We studied equine Cyt c variants in which these lysine residues are substituted. NMR spectra are shown in Figure 3. The Lys72Gly mutation has no significant effect on the alkaline transition (Table I), or on red foldon stability $(\mathrm{HX})$, or on protein stability (melting), or on protein structure [NMR; Fig. 3(A)], and the alkaline transition produces a normal low spin product (electronic spectrum). These results show that Lys 72 does not contribute significantly to the alkaline transition.

The additional mutation of Lys73 to Gly has little effect on the transition $\mathrm{pK}_{\mathrm{a}}$ (Table I) or spin state suggesting that Lys73 makes little contribution, which would be unlike results for yeast Cyt c. ${ }^{12}$ However, the mutant protein and the red loop are mildly destabilized, by 0.8 $\mathrm{kcal} / \mathrm{mol}$ ), evidently due to the loss of a stabilizing salt link 

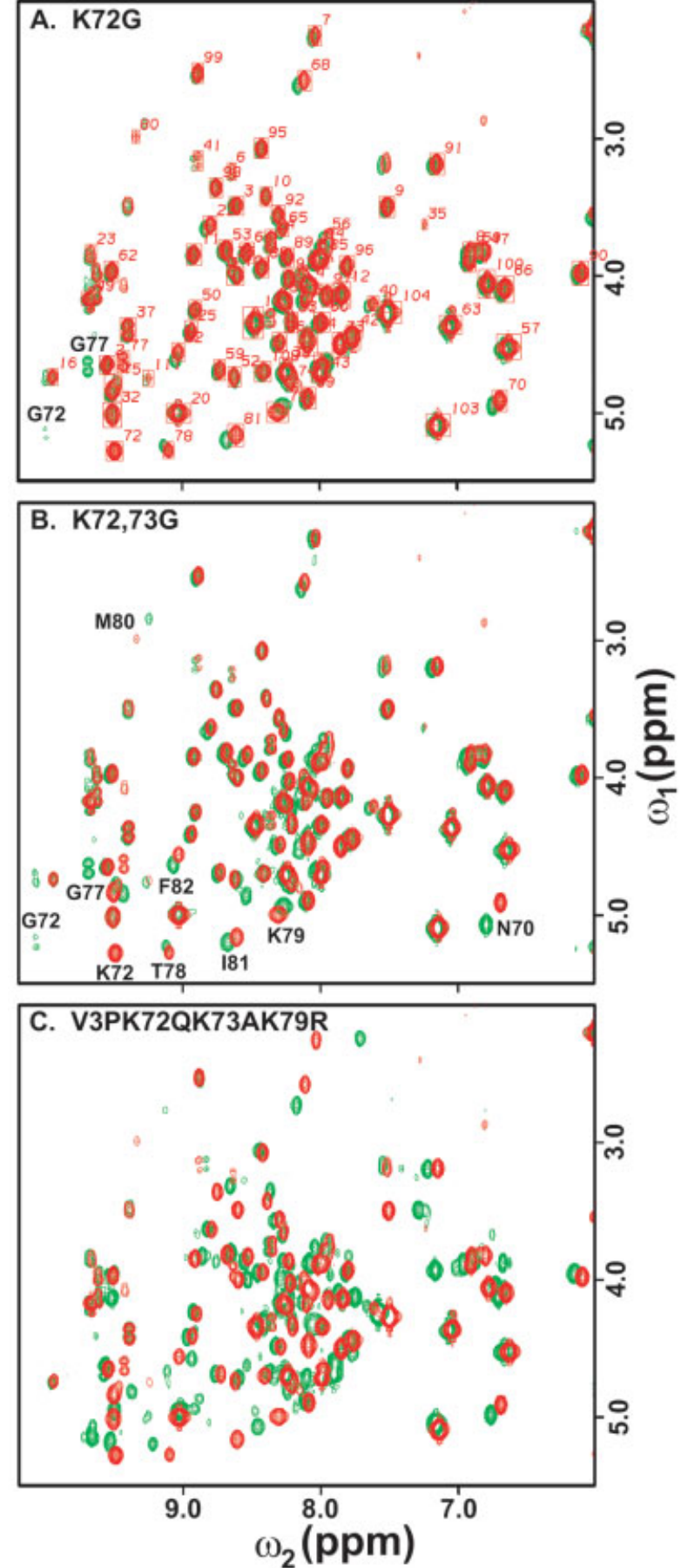

Fig. 3. NMR spectra. The fingerprint region of 2D-COSY spectra (NH-C ${ }_{\alpha} \mathrm{H}$ crosspeaks) for the parent pWT Cyt c (red) is compared with mutants in which specific lysines have been substituted (green). Lys72Gly (A) shows no changes (residue numbers shown). The additional Lys73Gly mutation causes changes limited to the red $\Omega$-loop (B, residues 70-85). The further Lys79Arg mutation (C) produces widespread changes.

to Glu66. Changes in NMR chemical shift are seen but only in the red loop (Fig. 3B). Given the $0.8 \mathrm{kcal} / \mathrm{mol}$ destabilization of the red loop, one could expect the alkaline transition to be promoted by 0.5 units in $\mathrm{pK}_{\mathrm{a}}$ if it were due to Lys79 misligation alone. Only 0.16 unit is found (Table I) suggesting that some other compensating effect tends to disfavor the transition and increase the $\mathrm{pK}_{\mathrm{a}}$. The obvious suggestion is that the disfavoring effect is the loss of Lys73 itself which does in fact act as a contributing ligand.

When Lys79 is additionally substituted (Lys79Arg), the transition $\mathrm{pK}_{\mathrm{a}}$ is raised but only from 9.16 to 9.8 (Table I). However, it turns out that the misligation in this case is due to the $\mathrm{N}$-terminal amino group which is not blocked in the recombinant pWT protein. When the $\mathrm{N}$-terminus is blocked by a pyroglutamate residue, ${ }^{17}$ the apparent $\mathrm{pK}_{\mathrm{a}}$ rises to 10.3 , and even then the $\mathrm{pH}$ titration is multiphasic and the misligated $\mathrm{Fe}$ is high spin, apparently due to $\mathrm{OH}^{-}$-ion ligation. These results show that Lys79 contributes to the alkaline transition, and apparently also Lys73, and they demonstrate the absence of any other candidate low spin ligand. Qualitatively, Lys79 appears to be the major contributor.

When Lys79 is substituted, major perturbations appear in the NMR spectrum [Fig. 3(C)]. These changes may be due to some significant local perturbation and/or to loss of the H-bond connecting Lys79- $\mathrm{NH}_{3}^{+}$to the Thr47 carbonyl. It is interesting that apparently benign mutations can produce widespread structural perturbations and also, as seen for other mutants, stability changes in some foldons that affect some functional property (e.g., the red loop and the alkaline transition). Previous interpretations of mutational effects on the alkaline transition have sometimes been misled by assuming only direct effects due to specific residue mutations.

\section{DISCUSSION}

The alkaline transition occurs universally in prokaryotic and eukaryotic ferricytochromes c. The transition has been long known ${ }^{28}$ and has been studied by many workers. ${ }^{29}$ The identity of the replacement ligand and of a putative trigger group have been of enduring interest.

Early work to test for the replacement ligand studied the alkaline transition in variants in which some or all of the lysine residues (15 in yeast; 19 in horse) were chemically modified (gathered in Table I of Rosell et al. ${ }^{12}$ ). The apparent transition $\mathrm{pK}_{\mathrm{a}}$ appeared to be only moderately increased (see Lys79 above). These results were variably interpreted to suggest the involvement in the transition of lysine or nonlysine residues, including some as yet unidentified "trigger" residue. The deprotonation of the trigger was thought to be necessary to drive some structural rearrangement that removes the Met80 ligand and translates in the replacement ligand. This view drew support from the observation in kinetic alkaline transition experiments ${ }^{30,31}$ of two spectrally observable steps, with some unidentified deprotonation reaction preceding the ligand replacement reaction (see also Martinez and Bowler ${ }^{32}$ ). The demonstration that the alkaline form of yeast Cyt $\mathrm{c}$ involves more than one NMR conformer, indicating different alternative ligands, was an important advance. ${ }^{33}$

The development by Mauk and coworkers ${ }^{16}$ of a recombinant system for cloning Cyt c mutants together with functional heme lyase made it possible to study specifically modified constructs, which has pointed to lysine 73 and 79 
as alternative replacement residues in the yeast protein. ${ }^{11,12}$ The present work used a modified construct to express defined mutants of equine Cyt c in E. coli. ${ }^{17}$ Results obtained bear on the structural determinants of the alkaline transition, the residues involved, and the trigger hypothesis.

\section{Ligand Replacement and Triggering}

The results obtained here confirm a major role for Lys79 as the replacement residue in the horse Cyt $\mathrm{c}$ alkaline transition. Mutational substitution of Lys79 abolishes the normal transition to a low spin complex, when Lys73 is not available. It appears that Lys73 contributes also.

Does the deprotonation of some still elusive trigger residue rather than the replacement ligand itself drive the structural rearrangement necessary for ligand exchange? Information that appeared to support the action of some trigger residue now appears less compelling than was earlier inferred. The apparently small effect on the transition $\mathrm{pK}_{\mathrm{a}}$ of blocking the various lysines in Cyt c can be explained in terms of major structural perturbations [Fig. $3(\mathrm{C})$ ] and the role of alternative lysines and alternative ligands (e.g., hydroxide ion ${ }^{12}$ ). The spectrally observable fast phase in kinetic alkaline transition experiments may or may not promote the transition, but some triggering role is clearly not required. The transition is an equilibrium reaction that will find the condition-dependent lowest free energy state (see below). The major red loop rearrangement necessary to allow the transition occurs spontaneously, rapidly $(\tau \sim 100 \mathrm{msec})$, and repeatedly ${ }^{8}$ and needs no $\mathrm{pH}$-dependent triggering impulse in a kinetic sense, although it is clear that any equilibrium destabilization of the red loop will promote the transition.

\section{The Alkaline Transition as a Multi-Group Competition}

The ligand-exchange equilibrium can be written in two steps, an initial deprotonation reaction followed by heme ligand exchange, as follows.

$$
\begin{aligned}
& \operatorname{Cytc}(\mathrm{Fe}-\mathrm{S}) \cdot \mathrm{LysNH}_{3}^{+} \stackrel{\mathrm{pK}_{\mathrm{a}}=11.4}{\rightleftarrows} \mathrm{Cyt} \mathrm{c}(\mathrm{Fe}-\mathrm{S}) \cdot \mathrm{LysNH}_{2} \\
& \stackrel{\mathrm{K}=10^{2.3}}{\rightleftarrows} \mathrm{Mis}-\mathrm{Cyt} \mathrm{c}\left(\mathrm{Fe} \cdot \mathrm{NH}_{2} \mathrm{Lys}\right)
\end{aligned}
$$

The equilibrium observed at any condition is determined by the difference in stabilization free energy between all of the energetic interactions that favor the neutral $\mathrm{pH}$ form (left) and all that favor the alkaline form (right). The overall ligand exchange equilibrium poises at a midpoint of $\mathrm{pH} 9.16$ in $\mathrm{pWT}$ equine Cyt c where $\Delta \mathrm{G}_{\mathrm{AT}}^{\mathrm{o}}=0$ and it can then be tipped in either direction by adjusting the $\mathrm{pH}$ to titrate the lysine protonation state or by other factors that affect the equilibrium.

A number of factors enter into both of the balancing reactions. In the initial titration reaction, the apparent deprotonation $\mathrm{pK}_{\mathrm{a}}$ for the replacement ligand can be evaluated from kinetic $\mathrm{pH}$ jump experiments as the $\mathrm{pH}$ at which the ligand exchange rate rises to half of its maximum value. , 30,31 The effective deprotonation $\mathrm{pK}_{\mathrm{a}}$ is 11.4 , higher than the usual $\mathrm{pK}_{\mathrm{a}} \sim 10.4$ for free lysine, apparently because the cationic form of Lys79 is stabilized by H-bonding to Thr47-carbonyl. ${ }^{34}$ This restraining bond is worth over $1 \mathrm{kcal} / \mathrm{mol}$ [Eq. (1)]. The net equilibrium alkaline transition $\mathrm{pK}_{\mathrm{a}}$ of 9.16 is much lower than the Lys $\mathrm{pK}_{\mathrm{a}}$ because the overall reaction is drawn forward by the subsequent misligation equilibrium which favors neutral lysine over Met80-S by a factor of $\sim 100\left(\Delta \Delta \mathrm{G}^{\circ} \sim 3\right.$ $\mathrm{kcal} / \mathrm{mol}$ ).

We find that the misligation reaction is disfavored by the red $\Omega$-loop foldon. The red loop has a stabilization free energy against unfolding of about $5 \mathrm{kcal} / \mathrm{mol}$ in the pertinent high $\mathrm{pH}$ range. ${ }^{8}$ [It is $6 \mathrm{kcal} / \mathrm{mol}$ at $\mathrm{pH} 7$; Fig. 2(B)]. Change in the stabilization free energy of the red loop (denaturant, mutations) appears quantitatively in the transition equilibrium, and this relationship is seen to remain linear for destabilizing factors that reach to almost half of the overall red loop stability [Fig. 2(D)]. Thus it appears that the red loop gives up most or all of its stabilization free energy in the transition. The stability of the red loop appears to be the largest factor in the calibration and control of the transition.

The design of the protein maintains a delicate balance between these large energies, with the strong misligation tendency of the vicinal Lys79 counterbalanced by its aberrantly high $\mathrm{pK}_{\mathrm{a}}$ and especially by the surprisingly large stability of the red loop. At pH 7.4 the net stability of the neutral form of Cyt c relative to the alkaline form is only $\sim 2 \mathrm{kcal} / \mathrm{mol}$ in free energy and it decreases in a continuous way with the internally controlled red loop stability as with the externally controlled $\mathrm{pH}$.

It is interesting to wonder why the protein places potentially misligating lysine residues so dangerously close to its active center. It has often been suggested that Cyt $\mathrm{c}$ might switch to the high $\mathrm{pH}$ form to adjust its reduction potential and so help to control its redox interactions in the intermembrane mitochondrial space. ${ }^{29}$ In this regard, it is interesting that the amino acid sequence of the red loop, seen here to control the alkaline transition, is almost totally conserved. Also a hydrogen exchange study suggestively shows that Cyt c does tend to partially unfold in the presence of lipid vesicles. ${ }^{35}$

\section{Conclusions}

A previous HX study indicated that the rate of the Cyt $\mathrm{c}$ alkaline transition is limited by the rate for concerted unfolding of an $\Omega$-loop foldon. ${ }^{8}$ The present work finds that the same foldon unit controls the alkaline transition equilibrium. Essentially the entire stabilization free energy of the foldon is lost in the structural distortion necessary to withdraw the normal ligand and present the replacement ligand to the heme iron. Structurally, the normal Met80 heme ligand in Cyt $\mathrm{c}$ and the replacement ligands in the alkaline transition, Lys79 and Lys73, are all carried by the same $\Omega$-loop foldon segment. The equilibrium character of the transition and the intrinsically dynamic property of foldons ensure that no additional mechanism is necessary to kinetically trigger the ligand exchange reaction. 
It seems likely that biological evolution has not overlooked the ability of dynamic foldon behavior to control the equilibrium and kinetic properties of other protein functional processes.

\section{ACKNOWLEDGMENTS}

This paper is dedicated to George Rose who first discovered $\Omega$-loops, documented their wide occurrence, and described their structural properties (with J. FetrowLeszczynski ${ }^{36}$ ). His dedication to scientific and human values continues to serve as a guiding influence. This work was supported by NIH research grant GM31847.

\section{REFERENCES}

1. Maity H, Maity M, Krishna MMG, Mayne L, Englander SW. Protein folding: the stepwise assembly of foldon units. Proc Natl Acad Sci USA 2005;102:4741-4746.

2. Bai Y, Sosnick TR, Mayne L, Englander SW. Protein folding intermediates: native-state hydrogen exchange. Science 1995;269: 192-197.

3. Chamberlain AK, Handel TM, Marqusee S. Detection of rare partially folded molecules in equilibrium with the native conformation of RNase H. Nature Struct Biol 1996;3:782-787.

4. Fuentes EJ, Wand AJ. Local dynamics and stability of apocytochrome $b_{562}$ examined by hydrogen exchange. Biochemistry 1998 ; 37:3687-3698.

5. Fuentes EJ, Wand AJ. Local stability and dynamics of apocytochrome $b_{562}$ examined by the dependence of hydrogen exchange on hydrostatic pressure. Biochemistry 1998;37:9877-9883.

6. Chu R, Pei W, Takei J, Bai Y. Relationship between the nativestate hydrogen exchange and folding pathways of a four-helix bundle protein. Biochemistry 2002;41:7998-8003.

7. Yan S, Kennedy S, Koide S. Thermodynamic and kinetic exploration of the energy landscape of Borrelia burgdorferi OspA by native-state hydrogen exchange. J Mol Biol 2002;323:363-375.

8. Hoang L, Maity H, Krishna MM, Lin Y, Englander SW. Folding units govern the cytochrome c alkaline transition. J Mol Biol 2003;331:37-43

9. Krishna MMG, Lin Y, Rumbley JN, Englander SW. Cooperative omega loops in cytochrome c: role in folding and function. J Mol Biol 2003;331:29-36.

10. Schechter E, Saludjian P. Conformation of ferricytochrome c. IV. Relationship between optical absorption and protein conformation. Biopolymers 1967;5:788-790.

11. Ferrer JC, Guillemette JG, Bogumil R, Inglis SC, Smith M, Mauk AG. Identification of Lys79 as an iron ligand in one form of alkaline yeast iso-1-ferricytochrome c. J Am Chem Soc 1993;115: $7507-7508$.

12. Rosell FI, Ferrer JC, Mauk AG. Proton-linked protein conformational switching: definition of the alkaline conformational transition of yeast Iso-1-ferricytochrome c. J Am Chem Soc 1998;120: 11234-11245.

13. Margoliash E, Frohwirt N. Spectrum of horse heart cytochrome c. Biochem J 1959;71:570-572.

14. Nozaki Y. The preparation of guanidinium chloride. Methods Enzymol 1972;26:43-50.

15. Maity H, Maity M, Englander SW. How cytochrome c folds, and why: submolecular foldon units and their stepwise sequential stabilization. J Mol Biol 2004;343:223-233.

16. Pollock WBR, Rosell FI, Twitchett MB, Dumont ME, Mauk AG. Bacterial expression of a mitochondrial cytochrome c. Trimethyla- tion of Lys72 in yeast iso-1-cytochrome c and the alkaline conformational transition. Biochemistry 1998;37:6124-6131.

17. Rumbley JN, Hoang L, Englander SW. Recombinant equine cytochrome c in Escherichia coli: High-level expression, characterization, and folding and assembly mutants. Biochemistry 2002;41: 13894-13901.

18. Cochran DA, Doig AJ. Effect of the N2 residue on the stability of the alpha-helix for all 20 amino acids. Protein Sci 1305;10:13051311.

19. Pace CN. Determination and analysis of urea and guanidine hydrochloride denaturation curves. Methods Enzymol 1986;131: $266-280$.

20. Santoro MM, Bolen DW. Unfolding free energy changes determined by the linear extrapolation method. 1. Unfolding of phenylmethanesulfonyl alpha-chymotrypsin using different denaturants. Biochemistry 1988;27:8063-8068.

21. Mayne L, Englander SW. Two-state vs. multi-state protein unfolding studied by optical melting and hydrogen exchange. Protein Sci 2000;9:1873-1877.

22. Eftink MR, Ionescu R. Thermodynamics of protein unfolding: questions pertinent to testing the validity of the two-state model. Biophys Chem 1997;64:175-197.

23. Bai Y, Milne JS, Mayne L, Englander SW. Primary structure effects on peptide group hydrogen exchange. Proteins 1993;17:7586 .

24. Connelly GP, Bai Y, Jeng MF, Englander SW. Isotope effects in peptide group hydrogen exchange. Proteins 1993;17:87-92.

25. Myers JK, Pace CN, Scholtz JM. Denaturant $m$ values and heat capacity changes: relation to changes in accessible surface areas of protein unfolding. Protein Sci 1995;4:2138-2148.

26. Nall BT, Zuniga EH, White TB, Wood LC, Ramdas L. Replacement of a conserved proline and the alkaline conformational change in iso-2-cytochrome c. Biochemistry 1989;28:9834-9839.

27. Xu Y, Mayne LC, Englander SW. Evidence for an unfolding and refolding pathway in cytochrome c. Nature Struct Biol 1998;5:774778 .

28. Theorell H, Åkesson A. Studies on cytochrome c. II. The optical properties of pure cytochrome $\mathrm{c}$ and some of tts derivatives. J Am Chem Soc 1941;63:1812-1818.

29. Wilson MT, Greenwood C. The alkaline transition in ferricytochrome c. In: Scott RA, Mauk AG, editors. cytochrome c: a multidisciplinary approach. Sausolito: University Science Books; 1996. p 611-634.

30. Davis LA, Schejter A, Hess GP. Alkaline isomerization of oxidized cytochrome c. Equilibrium and kinetic measurements. J Biol Chem 1974;249:2624-2632.

31. Kihara H, Saigo S, Nakatani H, Hiromi K, Ikeda-Saito M, Iizuka T. Kinetic study of isomerization of ferricytochrome $\mathrm{c}$ at alkaline pH. Biochim Biophys Acta 1976;430:225-243.

32. Martinez RE, Bowler BE. Proton-mediated dynamics of the alkaline conformational transition of yeast iso-1-cytochrome c. J Am Chem Soc 2004;126:6751-6758.

33. Hong XL, Dixon DW. NMR study of the alkaline isomerization of ferricytochrome c. FEBS Lett 1989;246:105-108.

34. Bushnell GW, Louie GV, Brayer GD. High-resolution three dimensional structure of horse heart cytochrome c. J Mol Biol 1990;213: $585-595$

35. Pinheiro TJ, Cheng H, Seeholzer SH, Roder H. Direct evidence for the cooperative unfolding of cytochrome $\mathrm{c}$ in lipid membranes from H-D exchange kinetics. J Mol Biol 2000;303:617-626.

36. Leszczynski JF, Rose GD. Loops in globular proteins: a novel category of secondary structure. Science 1986;234:849-855.

37. Kraulis PJ. MOLSCRIPT: a program to produce both detailed and schematic plots of proteins structures. J Appl Crystallogr 1991;24: 946-950. 